\title{
Hydrogel Expandable Coils for Vascular Occlusion in Congenital Cardiovascular Disease: A Single Center Experience
}

\author{
Bryan H. Goldstein, MD, Ranjit Aiyagari, MD, Martin L. Bocks, MD, and Aimee K. Armstrong, MD \\ Department of Pediatrics, C. S. Mott Children's Hospital, University of Michigan Health System, Ann Arbor, Mich, USA
}

\begin{abstract}
A B S T R A C T
Objective. The objective of this study is to evaluate the safety and feasibility of the AZUR hydrogel-polymer coated platinum coil (hydrocoil) for vascular occlusion in patients with congenital cardiovascular disease (CCVD).

Design. Retrospective case review.

Patients. Eight patients with CCVD who underwent attempted vascular occlusion procedure with the hydrocoil at the University of Michigan between January 1, 2008 and March 1, 2011. Median patient age was 1.6 years (range $0.6-27.5$ years) and median weight was $10.6 \mathrm{~kg}(3.9-74.8 \mathrm{~kg})$.

Outcome Measures. Complete vascular occlusion of the target vessel was assessed at procedural completion with angiography and noninvasive imaging at follow-up. Key procedural variables and adverse events were also evaluated. Results. A total of 21 hydrocoils were successfully placed in seven vessels, in six patients. Vessels included four (57\%) veno-venous collaterals, two (29\%) aortopulmonary collaterals, and one (14\%) main pulmonary artery. Hydrocoils could not be placed successfully in two patients, including one baffle leak and one veno-venous collateral. Pretreatment of the hydrocoil with steam to soften the coil, prior to vascular introduction, increased the likelihood of successful implant (100\% vs. $60 \%$ ). Complete occlusion was observed in $100 \%$ of vessels either at the time of catheterization or at follow-up. There were no complications attributable to the hydrocoil.

Conclusions. Hydrocoils are safe and effective devices that can be utilized for vascular occlusion in a variety of blood vessels in patients with CCVD. Coil pretreatment may increase the likelihood of procedural success.
\end{abstract}

Key Words. Congenital Heart Disease; Collaterals; Embolization; Coil; Pediatric Interventions

\section{Introduction}

$\mathrm{T}$ he armamentarium of materials and devices available to the pediatric interventional cardiologist for closure of unwanted congenital or acquired vascular connections has grown exponentially in recent years. Although once limited to a polyvinyl alcohol Ivalon plug, closure options now include pushable stainless steel coils, detachable coils of various materials, vascular plugs, embolization glue, microspheres, and others. ${ }^{1}$ The hydrogel-coated expandable embolization coil only recently has begun to be utilized in the field of congenital vascular occlusion, although it has been widely applied in the treatment of intracranial aneurysm starting in 2002. ${ }^{2-5}$

The AZUR peripheral hydrocoil embolization system (hydrocoil, Terumo Medical Corporation,
Somerset, NJ, USA) combines a hydrogel-coated platinum coil with a screwless proprietary delivery system, offering numerous advantages over traditional stainless steel pushable coils. The hydrocoil provides for improved volumetric filling of vessels, mechanical occlusion that does not depend upon thrombus formation, increased delivery control, and enhanced reconstruction of the arterial wall, including earlier neointimal formation and healing compared with noncoated coils. ${ }^{6-10}$ Platinum hydrocoils are available in detachable or pushable delivery forms, in $0.018^{\prime \prime}$ and $0.035^{\prime \prime}$ wire diameters, as helical or framing coils, and in a large number of lengths and loop diameters. Use of the hydrocoil in congenital cardiovascular disease has not been reported to date. We sought to describe our experience with the hydrocoil for vascular occlusion in congenital cardiovascular disease at the University of Michigan. 
Table 1. Demographic and Medical Characteristics of the Study Population

\begin{tabular}{llllll}
\hline $\begin{array}{l}\text { Patient } \\
\text { No. }\end{array}$ & Sex & $\begin{array}{l}\text { Age } \\
\text { (Years) }\end{array}$ & $\begin{array}{l}\text { Weight } \\
(\mathrm{Kg})\end{array}$ & Diagnosis & Indication \\
\hline 1 & $\mathrm{~F}$ & 27.5 & 53.7 & SVC occlusion & Cyanosis \\
2 & $\mathrm{~F}$ & 17.7 & 60.3 & Ebstein's s/p Fontan & Cyanosis \\
3 & $\mathrm{~F}$ & 0.6 & 7.2 & TOF/PA/MAPCA & Dual supply collateral \\
4 & $\mathrm{~F}$ & 1.8 & 12.3 & Tricuspid atresia s/p Fontan & Cyanosis \\
5 & $\mathrm{~F}$ & 0.2 & 3.9 & Heterotaxy, single ventricle physiology s/p PA & Pulmonary overcirculation \\
& & & & band and aortopulmonary shunt & Dual supply collateral \\
6 & $\mathrm{M}$ & 1.0 & 7.5 & TOF/PA/MAPCA & Cyanosis \\
7 & $\mathrm{M}$ & 14.5 & 74.8 & HLHS s/p Fontan & Cyanosis \\
8 & $\mathrm{M}$ & 1.4 & 8.9 & SS,L,L\}, VSD, CoA s/p Senning/arterial switch & \\
\hline
\end{tabular}

CoA, coarctation of the aorta; HLHS, hypoplastic left heart syndrome; PA, pulmonary artery; SVC, superior vena cava; TOF/PA/MAPCA, tetralogy of Fallot with pulmonary atresia and multiple aortopulmonary collateral arteries; VSD, ventricular septal defect; M, male; F, female; s/p, status post.

\section{Materials and Methods}

A query of the cardiac catheterization laboratory database identified all patients with congenital cardiovascular disease who underwent attempted vascular occlusion procedure with a hydrocoil between January 1, 2008 and March 1, 2011. Demographic and medical characteristics were identified and key procedural components were recorded, including target blood vessel characteristics, attempts at vascular occlusion with alternative device(s), hydrocoil pretreatment, and procedural success. All original angiograms were reviewed (BHG). Technical success was defined as complete occlusion at the time of the procedure or appropriate coil position with no embolization to, or obstruction of, nontarget vessels. Complete occlusion was defined as complete angiographic occlusion at the time of the procedure or if subsequent imaging demonstrated a lack of residual flow in the target vessel. Median time from coil placement to follow-up imaging study documenting complete vascular occlusion was determined, when complete occlusion was not achieved in the catheterization laboratory. Complications of the procedure were recorded.

Cardiac catheterizations were performed in routine fashion under procedural sedation or general anesthesia according to provider preference. Hydrocoil sizing was determined based upon target vessel minimal diameter at intended location of deployment, with the goal of placing a coil with diameter approximately equaling 1.5 to 2.5 times the target vessel minimal diameter. Pretreatment of the coil with steam was performed based upon the preference of the provider. Coil deployment was performed according to standard techniques.

\section{Results}

Eight patients met inclusion criteria for the study. Demographic and medical characteristics are detailed in Table 1. A total of 21 hydrocoils were placed in seven blood vessels, in six patients. All implanted hydrocoils were $0.018^{\prime \prime}$ in diameter. Hydrocoil implantation was not successfully performed in two patients. The median patient age was 1.6 years (range 0.6-27.5 years) and median weight was $10.6 \mathrm{~kg}$ (range 3.9-74.8 kg). Hydrocoil deployment was performed in a variety of blood vessels including venous collaterals (57\%), aortopulmonary collaterals (29\%), and other vessels (14\%). Unsuccessful implantations occurred in one veno-venous collateral and one Senning baffle leak.

Results of hydrocoil implantation are shown in Table 2. Technical success was achieved in all patients in whom a hydrocoil was released. Hydrocoil delivery resulted in complete occlusion in the catheterization laboratory in $67 \%$ of cases. Complete occlusion was achieved in the remaining $33 \%$ of cases at a median follow-up time of 8.3 months. No patients have demonstrated evidence of recanalization (defined by return of flow in the target vessel) by follow-up imaging studies. Therefore, complete vascular occlusion was achieved in $100 \%$ of patients who received a hydrocoil.

\section{Venous Collaterals}

A total of 16 hydrocoils were delivered in four vessels in three patients. Venous collateral vessels included three veno-venous collaterals and one veno-atrial collateral. All patients had single ventricle physiology and were at the post-Fontan stage. Complete occlusion was documented at catheterization in $75 \%$ of these venous collaterals. The remaining vessel was documented to be completely occluded by imaging at follow-up. Occlusion of a veno-venous collateral using a hydrocoil was attempted in one additional patient but the coil was not deployed because of failure to achieve 
Table 2. Results of Hydrocoil Vascular Occlusions

\begin{tabular}{|c|c|c|c|c|c|c|}
\hline $\begin{array}{l}\text { Patient } \\
\text { No. }\end{array}$ & $\begin{array}{l}\text { Target } \\
\text { Vessel }\end{array}$ & $\begin{array}{l}\text { Vessel } \\
\text { Diameter (mm) }\end{array}$ & $\begin{array}{l}\text { Coil Loop } \\
\text { Diameter(s) }(\mathrm{mm})\end{array}$ & $\begin{array}{l}\text { No. of Coils } \\
\text { Implanted }\end{array}$ & $\begin{array}{l}\text { Coil } \\
\text { Pretreated }\end{array}$ & $\begin{array}{l}\mathrm{CO} \text { on } \\
\text { Angio or } \mathrm{F} / \mathrm{u}\end{array}$ \\
\hline 1 & VVC & 1.7 & 3 & 0 & No & $\mathrm{N} / \mathrm{A}$ \\
\hline 3 & APC & 1.8 & 4 & 1 & Yes & Yes \\
\hline 4 & VVC & 2.6 & 4 & 2 & Yes & Yes \\
\hline 5 & MPA & 4.0 & 4 & 3 & Yes & Yes \\
\hline 6 & APC & 1.5 & 4 & 1 & No & Yes \\
\hline 7 & VVC & 6.3 & 8,10 & 3 & No & Yes \\
\hline 8 & Baffle leak & 4.5 & 10 & 0 & No & $\mathrm{N} / \mathrm{A}$ \\
\hline
\end{tabular}

Angio, angiogram; APC, aortopulmonary collateral; CO, complete occlusion of target vessel; F/u, follow-up noninvasive imaging; MPA, main pulmonary artery; Vessel diameter, minimal diameter of target vessel; VVC, veno-venous collateral; N/A, not applicable.
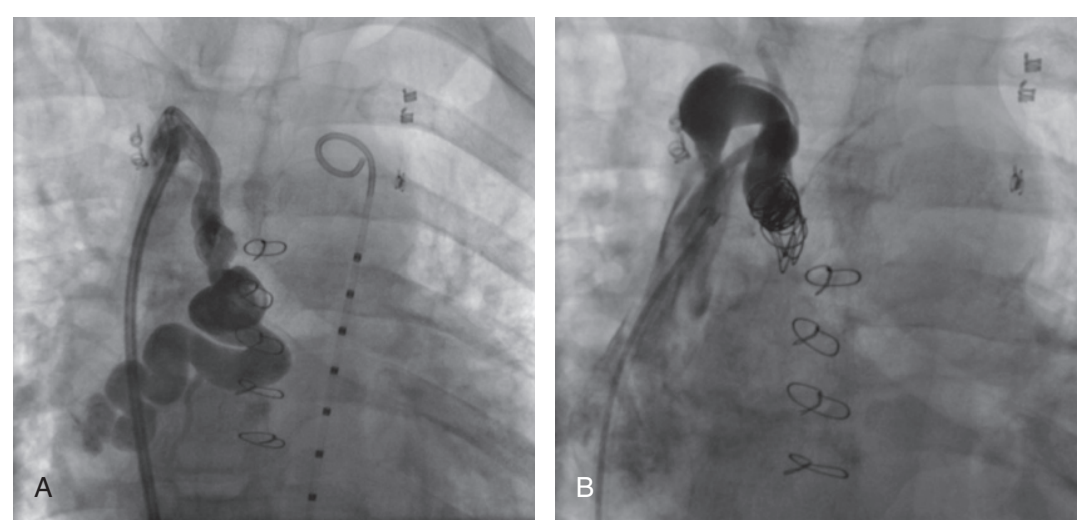

Figure 1. Large and tortuous veno-venous collateral in a 14-year-old patient with hypoplastic left heart syndrome palliated to Fontan physiology, who presented with increased cyanosis. Panel A shows the collateral in the anteroposterior projection, prior to intervention. Panel B demonstrates complete occlusion of the veno-venous collateral following deployment of three hydrocoils.

the desired coil confirmation. Figure 1 demonstrates a large tortuous veno-venous collateral that has been completely occluded following placement of three hydrocoils in a 14-year-old male with hypoplastic left heart syndrome palliated to Fontan physiology.

\section{Aortopulmonary Collaterals}

A total of two hydrocoils were delivered in two vessels in two patients. Both patients had tetralogy of Fallot with pulmonary atresia and multiple aortopulmonary collateral vessels. Complete occlusion was documented at catheterization in one of these aortopulmonary collaterals. The remaining vessel was documented to be completely occluded by imaging at follow-up. Figure 2 demonstrates an aortopulmonary collateral that has been completely occluded following placement of a single hydrocoil in a 6-month-old female with tetralogy of Fallot, pulmonary atresia, and multiple aortopulmonary collateral arteries status post unifocalization procedure.

\section{Other Vessels}

Two additional vascular occlusion procedures were undertaken using the hydrocoil. In one case, closure of a baffle leak with associated right-toleft shunt was attempted in a 16-month-old patient with $(\mathrm{S}, \mathrm{L}, \mathrm{L})$ transposition, ventricular septal defect, and coarctation status post coarctation repair, ventricular septal defect closure, Senning baffle, and arterial switch operation. Although the coil could be delivered to the desired location, its relatively straight conformation (this deployment was prior to our experience with coil pretreatment), floppy character, and location immediately adjacent to the mitral valve precluded the operators from safely deploying the hydrocoil. In the other case, definitive occlusion of a banded main pulmonary artery was attempted following the development of transfusion-dependent hemolysis after deployment of a pushable stainless steel Gianturco coil (Cook Medical Inc., Bloomington, IN, USA) in a 2-month-old with complex heterotaxy syndrome and single ventricle physiology 

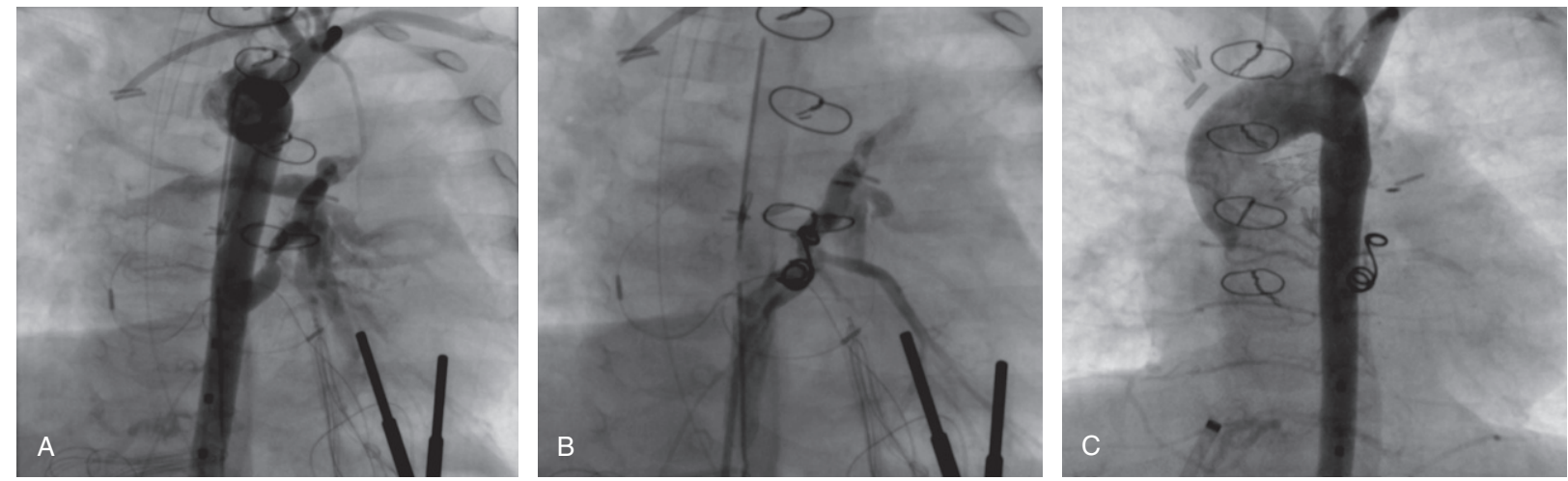

Figure 2. Aortopulmonary collateral artery in a 6-month-old patient with tetralogy of Fallot, pulmonary atresia, and multiple aortopulmonary collaterals, following unifocalization of important single-supply collateral vessels. Panel A depicts the aortopulmonary collateral arising from the descending aorta and connecting to the left lower lobe pulmonary artery, prior to intervention. In panel B, a single hydrocoil has been delivered with reduced but persistent aortopulmonary collateral flow. At catheterization 8 months later (panel $\mathrm{C}$ ), following right ventricle to pulmonary artery conduit implantation, no evidence of persistent collateral flow is identified in an aortogram.
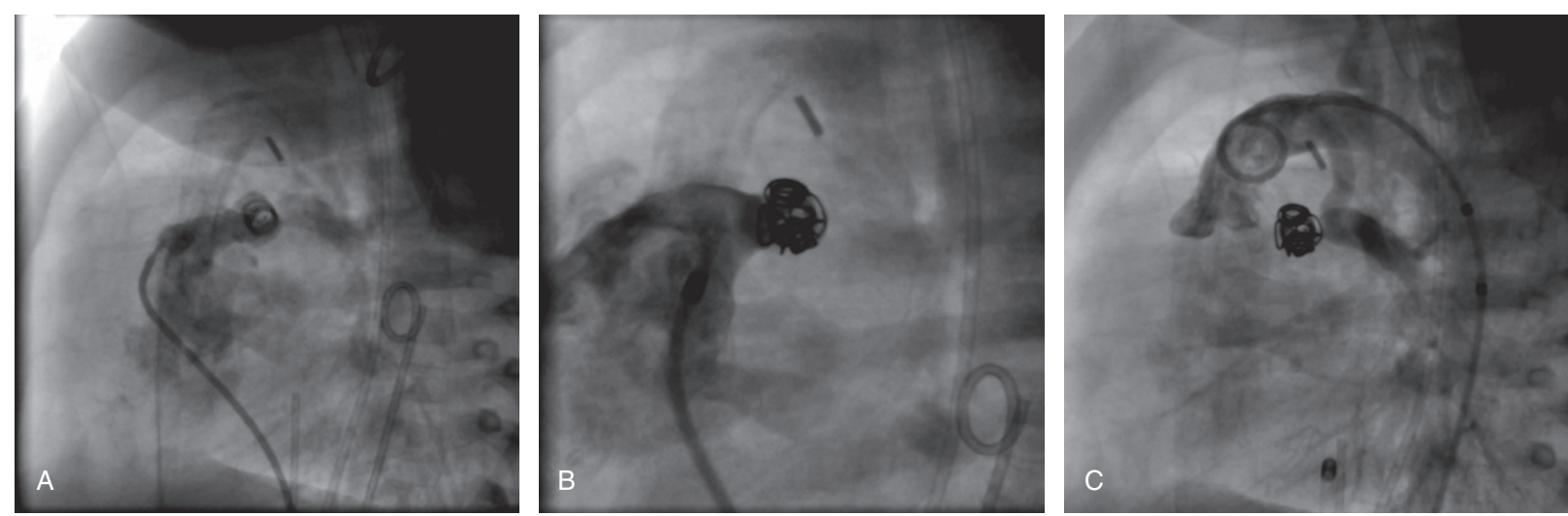

Figure 3. Main pulmonary artery in a 2-month-old patient with complex single ventricle anatomy status post pulmonary artery band (subtotal pulmonary artery ligation) and aortopulmonary shunt placement. Following the development of pulmonary overcirculation, a single pushable stainless steel Gianturco coil was deployed within the region between the stenotic native pulmonary valve annulus and the main pulmonary artery band, resulting in reduced but not eliminated blood flow (panel A). However, because of the development of transfusion-dependent hemolysis, the patient returned to the catheterization laboratory for placement of three hydrocoils, which resulted in complete vascular occlusion and cessation of active hemolysis (panels B \& C).

status post main pulmonary artery banding (subtotal pulmonary artery ligation) and aortopulmonary shunt placement. In this case, three hydrocoils were deployed in the main pulmonary artery, between the pulmonary valve and surgical band, nesting within the space partially occupied by the existing stainless steel coil (Figure 3). Complete vascular occlusion was observed angiographically and hemolysis later resolved.

\section{Coil Pretreatment}

In some instances, the hydrocoil was "pretreated" with steam to promote coil softening and reacqui- sition of a helical shape prior to exposure to the blood stream (Figure 4). This process allows the hydrocoil to assume immediately its intended "tight" helical conformation during deployment rather than to be delivered as a "loose" helical coil, assuming a tight helical conformation once in the vascular space for several minutes. We felt that pretreatment was particularly advantageous in cases in which the target vessel was small, short, or tortuous and in which the margin for error with coil delivery was small. Successful hydrocoil implantation occurred in $100 \%$ of cases in which a pretreatment strategy was employed and in $60 \%$ 

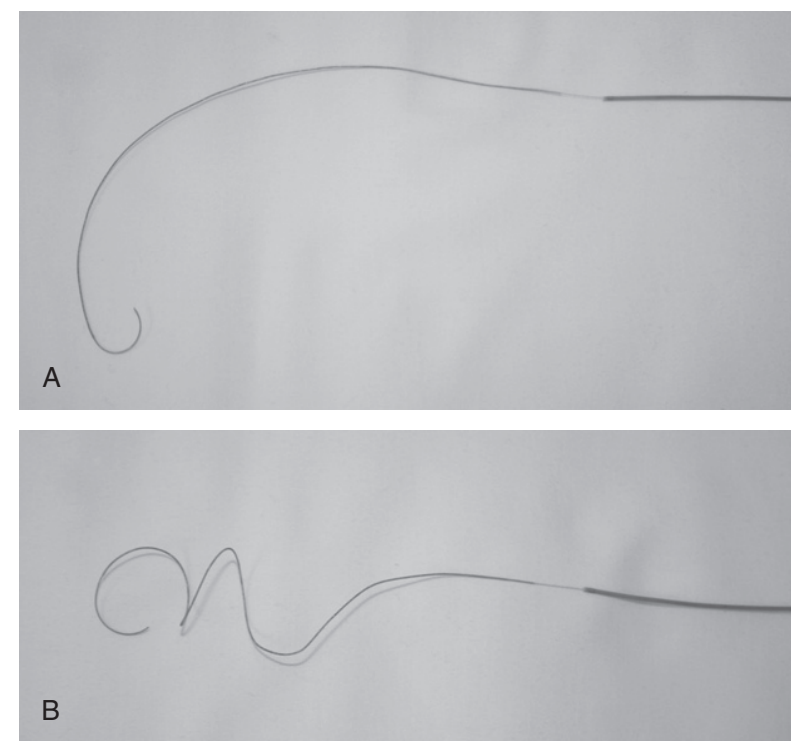

Figure 4. Effect of coil pretreatment with steam on coil conformation prior to vascular introduction. Panel A demonstrates a hydrocoil advanced via a microcatheter without any pretreatment, as it would be used "off the shelf." In panel $B$, the coil has been treated with steam prior to introduction into the microcatheter, allowing it to assume a more helical conformation.

of cases in which the hydrocoil was implanted as it comes "off the shelf."

\section{Complications}

There were no periprocedural complications in the eight patients in whom hydrocoil implantation was attempted. There were no coil embolizations to nontarget vessels, obstruction of nontarget vessels, or hemolysis.

\section{Discussion}

Although hydrogel-coated platinum coils have been used by interventional radiologists and neuroradiologists for nearly a decade, their use in congenital cardiovascular disease has not been described previously. In this report, we describe the implantation of hydrocoils in a wide range of patient ages, congenital heart defects, and target vessels, including venous collaterals, aortopulmonary collaterals, and other structures. We encountered no complications and had a 100\% target vessel complete occlusion rate when combining procedural angiographic and follow-up noninvasive imaging end points.

Recently, a number of reports have been published verifying the efficacy of newly introduced occlusion devices in the field of congenital cardiovascular disease, including the Amplatzer ductal occluder and vascular plug series (AGA Medical Corporation, Plymouth, MN, USA). ${ }^{11-13}$ The Amplatzer vascular plugs I \& II have been shown to deliver high rates of complete vascular occlusion in a variety of target vessels without major complications. ${ }^{12,13}$ The second generation vascular plug was shown to be particularly useful for occlusion of high-flow tubular lesions, such as patent ductus arteriosus, aortopulmonary collaterals, and arteriovenous malformations. ${ }^{13}$ In contrast to the vascular plug, which requires either a stiff guide catheter or long sheath for delivery, the hydrocoil may be delivered through a microcatheter, enabling access to more tortuous and difficult to reach target vessels. The hydrogel coating allows a $0.018^{\prime \prime}$ diameter coil to provide greater volumetric filling, once fully hydrated, than a standard $0.035^{\prime \prime}$ diameter coil (approximately equivalent to a $0.04^{\prime \prime}$ diameter coil). Thus, the hydrocoil is particularly effective in occlusion of vessels that are difficult to access with a catheter or sheath large enough through which to deliver most occluding devices (such as the vascular plug or a $0.035^{\prime \prime}$ diameter coil). In our series, there were several cases in which vascular occlusion could not have been efficiently achieved (if occlusion could have been achieved at all) without the ability to deliver the hydrocoil(s) via a microcatheter.

A second significant advantage of the hydrocoil is the control with which it can be delivered, repositioned, and released. The delivery "wire" and platinum hydrocoil are one-piece "off the shelf" with no profile change at the connection site. The densely radio-opaque nature of the platinum coil makes the connection site obvious on fluoroscopy. Release of the hydrocoil is achieved almost instantaneously upon activation of a proprietary "handle" which delivers a small electric current via the delivery wire to the wire-coil connection site and facilitates a local electrolytic reaction. This degree of delivery control accounted for the lack of coil embolization or other device complications encountered in our series. Although our series is small, the $0 \%$ complication rate is far below that reported in previous large series of coil- and device-associated transcatheter vascular occlusion procedures. ${ }^{11,14,15}$

There are several other important advantages of the hydrocoil as a vascular occlusion device. As a platinum-based coil, the hydrocoil does not generate significant artifact on magnetic resonance 
imaging, a major drawback of the stainless steel coil in the current imaging era. ${ }^{16}$ Meanwhile, to date, there are no reports in the literature associating the hydrocoil with intravascular hemolysis. In fact, in our series, the hydrocoil was utilized to successfully treat ongoing transfusion-dependent hemolysis by completely occluding a main pulmonary artery that had been partially occluded with a Gianturco stainless steel coil. Additionally, we noted the development of late complete occlusion in one patient with an aortopulmonary collateral and incomplete angiographic occlusion at hydrocoil implantation. We believe this is due to continued coil hydration, beyond the initial 15-20 minutes of rapid coil hydration upon exposure to the bloodstream, and is an added benefit of the hydrocoil, when compared with a standard stainless steel coil. Moreover, the modality of hydrocoil vascular occlusion-mechanical (volumetric) luminal obstruction, rather than thromboocclusion-ought to lower the risk of postprocedural thromboembolism, compared with alternative methods of vascular occlusion.,

One potential drawback of the hydrocoil is the relatively stiff nature of the coil in its dehydrated form (off the shelf) prior to exposure to the bloodstream. This relatively stiff and straight nature of the coil could preclude successful delivery and implantation in small and tortuous target vessels, but ex vivo prehydration of the hydrocoil with steam modifies the hydrocoil such that it assumes its ultimate helical shape (see Figure 4). The coil is then loaded into the delivery catheter and assumes a tight helical shape immediately upon advancement into the vessel. Pretreatment does not risk overhydration of the hydrogel coating; the $0.018^{\prime \prime}$ coil can always be introduced into a microcatheter after this process. In fact, in our experience, the $0.018^{\prime \prime}$ hydrocoil could still be removed from the body via a microcatheter after coil pretreatment and an additional 5-10 minutes of hydration during coil positioning within the target vessel. Although we did not experience difficulty with coil removal via a microcatheter, the hydrocoil eventually becomes hydrated beyond a diameter at which removal through the microcatheter can be accomplished. This can happen as early as 10-15 minutes after exposure to the bloodstream. If this does happen, however, the hydrocoil and microcatheter combination can be removed together, via the guiding catheter, to facilitate successful removal of an unreleased hydrocoil.

In comparing devices for vascular occlusion, it is important to evaluate relative device costs.
While the stainless steel Gianturco coil will always be less expensive than newly introduced more complex and technically advanced devices, this comparison is probably less relevant in the present era. Indeed, when comparing the hydrocoil with other vascular occlusion devices approved in the last decade, the hydrocoil is equivalent in cost to the Amplatzer vascular plug II, well less expensive than the Amplatzer ductal occluder and slightly more expensive than the Flipper detachable coil (Cook Medical Inc., Bloomington, IN, USA).

\section{Conclusions}

In summary, we found hydrocoils to be safe and effective devices for vascular occlusion in a variety of circumstances routinely encountered in the treatment of patients with congenital cardiovascular disease. Hydrocoils offer several important advantages over existing occlusion devices including smaller delivery profile, enhanced delivery control, improved volumetric filling of vessels, earlier vascular healing, and decreased risk for hemolysis. Hydrocoil pretreatment may increase the likelihood of successful coil deployment. While not ideal for all target vessels, the hydrocoil significantly augments the existing vascular occlusion "tool set" of the pediatric interventional cardiologist.

Corresponding Author: Bryan H. Goldstein, MD, Cincinnati Children's Hospital Medical Center, Division of Cardiology, 3333 Burnet Avenue, ML 2003, Cincinnati, OH 45229, USA. Tel: (+1) 513-636-7072; Fax: (+1) 513-636-2410; E-mail: bryan.goldstein@ cchmc.org

Conflict of interest: The authors have no financial relationships with industry.

Accepted in final form: August 17, 2011.

\section{References}

1 Porstmann W, Wierny L, Warnke H. Closure of persistent ductus arteriosus without thoracotomy. Ger Med Mon. 1967;12:259-261.

2 White PM, Lewis SC, Nahser H, Sellar RJ, Goddard T, Gholkar A. HydroCoil Endovascular Aneurysm Occlusion and Packing Study (HELPS trial): procedural safety and operator-assessed efficacy results. A7NR Am $\mathcal{F}$ Neuroradiol. 2008;29:217-223.

3 Cloft HJ, Kallmes DF. Aneurysm packing with HydroCoil Embolic System versus platinum coils: initial clinical experience. A7NR Am 7 Neuroradiol. 2004;25:60-62. 
4 Abdel-Aal AK, Osman S, Hamed MF, Saddekni S, Saad WE. Embolization of bleeding duodenal ulcer using amplatzer vascular plug II and hydrogel coils: case report. Vasc Endovascular Surg. 2011;45:307310.

5 Gunnarsson T, Tong FC, Klurfan P, Cawley CM, Dion JE. Angiographic and clinical outcomes in 200 consecutive patients with cerebral aneurysm treated with hydrogel-coated coils. A7NR Am 7 Neuroradiol. 2009;30:1657-1664.

6 Sluzewski M, van Rooij WJ, Slob MJ, Bescos JO, Slump CH, Wijnalda D. Relation between aneurysm volume, packing, and compaction in 145 cerebral aneurysms treated with coils. Radiology. 2004;231:653-658.

7 Killer M, Arthur AS, Barr JD, Richling B, Cruise GM. Histomorphology of thrombus organization, neointima formation, and foreign body response in retrieved human aneurysms treated with hydrocoil devices. 7 Biomed Mater Res B Appl Biomater. 2010;94:486-492.

8 Ding YH, Dai D, Lewis DA, Cloft HJ, Kallmes DF. Angiographic and histologic analysis of experimental aneurysms embolized with platinum coils, Matrix, and HydroCoil. AfNR Am 7 Neuroradiol. 2005;26:1757-1763.

9 Kallmes DF, Fujiwara NH. New expandable hydrogel-platinum coil hybrid device for aneurysm embolization. AfNR Am 7 Neuroradiol. 2002;23: $1580-1588$.

10 O'Hare AM, Fanning NF, Ti JP, Dunne R, Brennan PR, Thornton JM. HydroCoils, occlusion rates, and outcomes: a large single-center study. AfNR Am $\mathcal{F}$ Neuroradiol. 2010;31:1917-1922.

11 Pass RH, Hijazi Z, Hsu DT, Lewis V, Hellenbrand WE. Multicenter USA Amplatzer patent ductus arteriosus occlusion device trial: initial and one-year results. 7 Am Coll Cardiol. 2004;44:513519.

12 Hill SL, Hijazi ZM, Hellenbrand WE, Cheatham JP. Evaluation of the AMPLATZER vascular plug for embolization of peripheral vascular malformations associated with congenital heart disease. Catheter Cardiovasc Interv. 2006;67:113-119.

13 Schwartz M, Glatz AC, Rome JJ, Gillespie MJ. The Amplatzer vascular plug and Amplatzer vascular plug II for vascular occlusion procedures in 50 patients with congenital cardiovascular disease. Catheter Cardiovasc Interv. 2010;76:411-417.

14 Brunetti MA, Ringel R, Owada C, et al. Percutaneous closure of patent ductus arteriosus: a multiinstitutional registry comparing multiple devices. Catheter Cardiovasc Interv. 2010;76:696-702.

15 Gudausky TM, Hirsch R, Khoury PR, Beekman $\mathrm{RH}$, III. Comparison of two transcatheter device strategies for occlusion of the patent ductus arteriosus. Catheter Cardiovasc Interv. 2008;72:675680.

16 Grifka RG, Fenrich AL, Tapio JB. Transcatheter closure of patent ductus arteriosus and aortopulmonary vessels using non-ferromagnetic Inconel MReye embolization coils. Catheter Cardiovasc Interv. 2008;72:691-695. 